\title{
An analytical near wall solution for the $\kappa-\epsilon$ model for transpired boundary layer flows
}

\author{
M.R. Avelino ${ }^{\mathrm{a}, 1}$, J. Su ${ }^{\mathrm{b}}$, A.P. Silva Freire ${ }^{\mathrm{a}, *}$ \\ ${ }^{a}$ Mechanical Engineering Program (PEM/COPPE/UFRJ), C.P. 68503, 21945-970, Rio de Janeiro, Brazil \\ ${ }^{\mathrm{b}}$ Nuclear Engineering Program (PEN/COPPE/UFRJ), C.P. 68509, 21945-970, Rio de Janeiro, Brazil
}

Received 7 May 1998; received in revised form 1 December 1998

\begin{abstract}
The present work uses asymptotic techniques to find analytical solutions for the velocity profile, the turbulent kinetic energy, $\kappa$, and the dissipation rate by unit mass, $\epsilon$, for transpired incompressible boundary layers. The higher-order solutions have an explicit dependence on the transpiration rate and can be used as wall functions for the specification of boundary conditions in the fully turbulent region of the flow. The second-order solutions show that $\kappa$ has a $(\ln y)$ behaviour whereas $\epsilon$ has a $(\ln y) / y$ behaviour. All results are validated against experimental data. (C) 1999 Elsevier Science Ltd. All rights reserved.
\end{abstract}

\section{Introduction}

A classical means of controlling the properties of turbulent boundary layers is the promotion of transpiration at the wall. With this artifice, momentum and heat can be added to the flow so that it can be made to conform to some desired property. For example, one may want to thicken the boundary layer, reduce the skin-friction, inhibit the transfer of heat at the wall, or avoid separation; all these flow features can easily be achieved by the injection or suction of fluid at the wall at controlled rates.

The perspectives opened by this means of controlling the properties of boundary layers raised great interest on the subject resulting in many experimental and theoretical contributions. A fair expectation, therefore,

\footnotetext{
* Corresponding author..

${ }^{1}$ Present address: Department of Mechanical Engineering, Rio de Janeiro State University (UERJ), Rua São Francisco Xavier 524, Maracanã, Rio de Janeiro, Brazil
}

is that, by now, the subject should have been largely understood with very few results to be accomplished. In fact, the progress achieved in the description of transpired turbulent flows in the last forty years was remarkable. Since the early studies on the subject by Mickley [1], passing through the very extensive experimental and theoretical works on incompressible flows by the Stanford Heat and Mass Transfer Group, and on compressible flows by the Cambridge University Aeronautics Division Group, much has been accomplished. The roles of transfer of heat, adverse and favourable pressure gradients, roughness, curvature, tri-dimensionality, compressibility, all have been studied in connection with transpired flow. As a consequence, some results have become firmly established. The existence of a logarithmic law of the wall for transpired flows is today an undisputed fact. Likewise, several turbulence models have been developed which provide good numerical predictions for some flow geometry. The flow conditions covered by the published experimental works have a wide spectrum, yielding data for many turbulent flow parameters of interest. In 


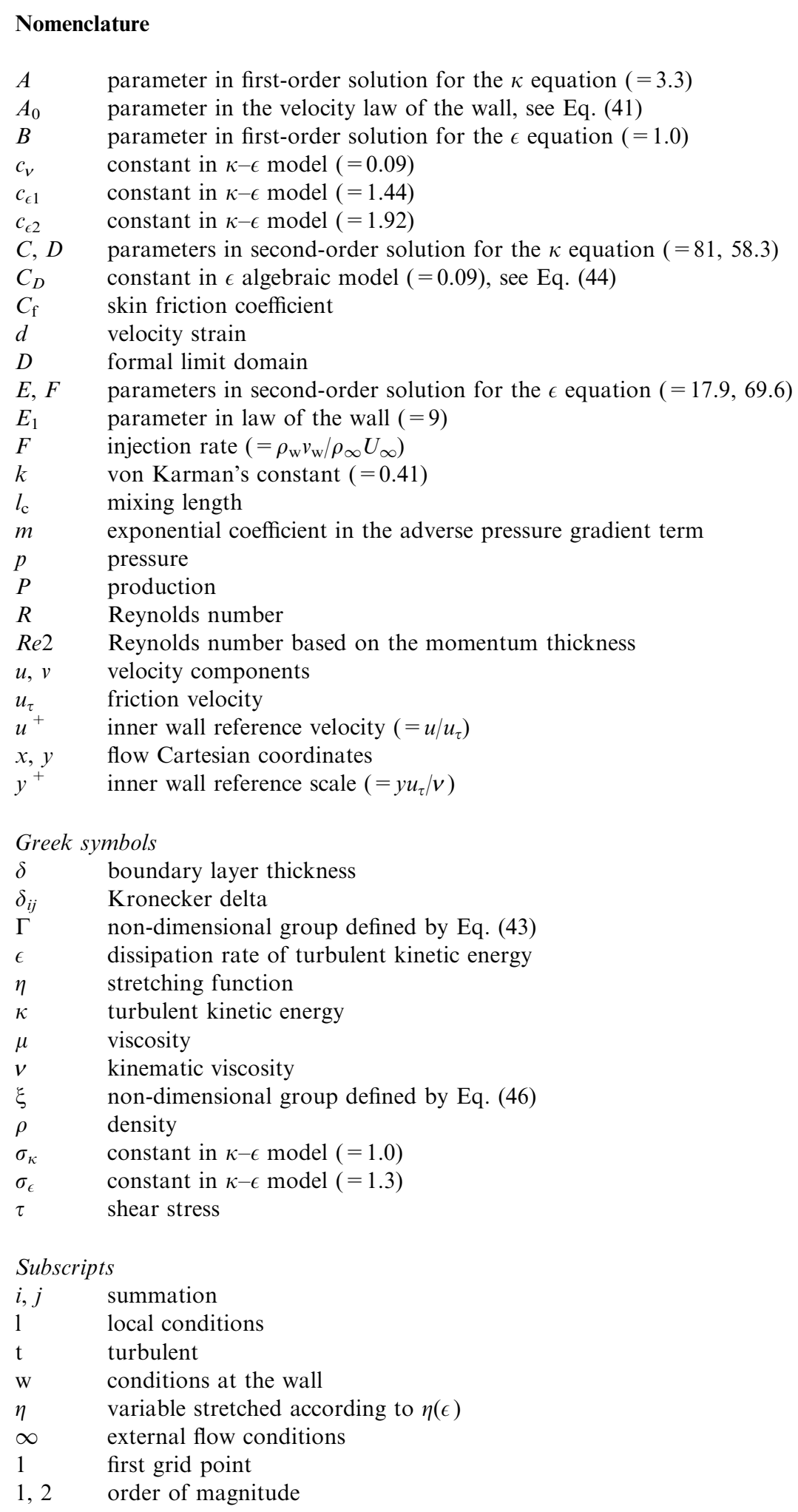




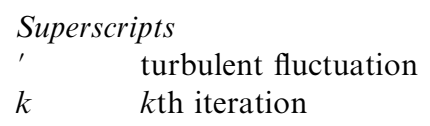

addition to the classical data on mean velocity and temperature profiles, and on some global parameters such as the skin-friction coefficient and the Stanton number, the works are relatively rich in turbulence data.

Despite all the achievements, some results still remain to be found. The analytical treatment of problems that combine compressibility effects, transfer of heat and transpiration, for instance, is extremely complex, having only recently been carried out [2]. Likewise, the study of the topology of a turbulent boundary layer with and without wall suction has recently been carried out by Antonia et al. [3]. The same authors were also involved in investigations on the effect of wall suction on bursting in a turbulent boundary layer [4] and on normalization for a turbulent boundary layer with wall transpiration [5].

A serious difficulty which always confronts all approaches, theoretical or experimental, is the correct description of flows subjected to very high injection rates. The uncertainty involved in the calculation of the skin-friction coefficient is a classical example. For instance, Kendall [6] have shown how sensitive to some flow parameters $C_{\mathrm{f}}$ is when obtained from any form of the momentum equation. They showed that for a transpiration rate of 0.005 at $R_{x}=10^{6}$, an uncertainty of $\pm 1 \%$ in the knowledge of both the momentum thickness variation with the distance from the leading edge and of the transpiration rate yields an uncertainty of $\pm 32 \%$ in $C_{\mathrm{f}}$. For transpiration rates of the order of 0.008 the boundary layer is said to be in a near 'blown-off' state yielding flow characteristics very difficult to measure and to reproduce theoretically.

The purpose of the present work is to develop a theory capable of successfully dealing with very high injection rates while thus presenting a large degree of generality. For that matter, we will choose as our turbulence closure model the $\kappa-\epsilon$ model. The present approach will resort to asymptotic techniques so as to find closed explicit analytical solutions for the flow velocity, $u$, turbulent kinetic energy, $\kappa$, and energy dissipation rate per unit mass, $\epsilon$, in the near wall fully turbulent region. These analytical solutions offer a convenient set of boundary conditions for a numerical computation of the flow field that avoids the complexities of the sublayer region. In this case, the outer region numerical solution is matched to the analytical solutions rather than to the conditions at the wall itself.

For flows where the near wall processes are too complex for the specification of analytical local equi- librium boundary conditions the remedy is to extend any specific turbulence model to the wall so that the viscous effects can be correctly captured. The different modelling strategies can, therefore, be grouped into those that strive at finding refined analytical solutions for the near wall solution and those that discard them in favour of a sophisticated modelling of the turbulent diffusivities across the near wall viscous sublayer.

An advantage of the low Reynolds number $\kappa-\epsilon$ models is that, in principle, they do not need any special treatment to deal with transpiration. Unfortunately, the predictions with such models provide too large values for the friction coefficient [7].

Another approach that dispenses a particular treatment of the wall conditions is the second moment closure of Launder and Shima [8]. This model is supposed to have a large degree of generality, being capable of accounting for non-equilibrium effects. Four cases of transpired flows are illustrated in Launder and Shima [8]. The agreement in the predicted values of $C_{\mathrm{f}}$ is very good for the test case with zero pressure gradient and moderate injection rates, whereas for the adverse pressure gradient case the agreement is poor.

The approaches that resort to a near wall analytical treatment of the solution normally consider the velocity profile to be given close to the wall by universal logarithmic laws. In this case, the turbulent kinetic energy is made directly proportional to the square of the local friction velocity and the dissipation rate directly proportional to the cube of the local friction velocity and inversely proportional to the distance from the wall. For transpired flows, these approximations are reasonable provided the transportation rate is small and the changes in the friction velocity are not significant. For high injection rates, modifications in the classical formulation must be made so as to correctly capture the strong explicit dependence of the wall region flow solution on the injection or suction velocity. In fact, as we shall see later, for high injection rates the dominance of the term which explicitly depends on the injection velocity on the flow solution is complete.

Perturbation techniques, which include some scaling arguments and hypotheses about the appropriate nature of asymptotic expansions for $u, \kappa$ and $\epsilon$, are used to find local approximated equations, that are then analytically solved. Important results found here are the second-order solutions which show that, if appropriate similarity parameters are used, the turbulent kinetic energy has a $(\log y)$ behaviour whereas the 
dissipation rate has a $(\log y) / y$ behaviour. The theory, as presented, is self-consistent, which means that all derived flow constants can be directly determined from the standard $\kappa-\epsilon$ constants. The obtained results can then be used to generate computed data for velocity profiles and skin-friction which can be compared with the experimental data of Andersen et al. [9]. An independent check on the theory can be made through a direct comparison of the high-order solutions with the turbulence data. The agreement shown by the theory is remarkable.

\section{The equations of motion}

We will now present the governing equations of the standard $\kappa-\epsilon$ formulation. Following the regular procedure, we denote the mean parts of the velocity and pressure fields by $u_{i}$ and $p$, respectively. The fluctuation of the turbulent quantities is denoted by the dashes.

Then, the equations of motion for incompressible flows can be cast as

$$
\frac{\partial u_{i}}{\partial x_{i}}=0
$$

$\frac{\partial u_{i}}{\partial t}+u_{j} \frac{\partial u_{i}}{\partial x_{j}}=-\frac{1}{\rho} \frac{\partial p}{\partial x_{i}}+\frac{\partial \tau_{i j}}{\partial x_{j}}+\frac{\partial}{\partial x_{j}}\left(v d_{i j}\right)$.

Here, $\rho$ is the density of the fluid. The Reynolds stress, $\tau_{i j}$, is

$\tau_{i j}=-\left\langle u_{i}^{\prime} u_{j}^{\prime}\right\rangle$

where the Dirac brackets denote time averaging. A summation is understood for repeated subscripts.

The standard $\kappa-\epsilon$ model relates the components of the Reynolds stress tensor to the mean flow gradients with the aid of the eddy viscosity concept

$\tau_{i j}=-\frac{2}{3} \kappa \delta_{i j}+v_{\mathrm{t}} d_{i j}$

Here, $\delta_{i j}$ is the Kronecker delta; $\kappa$, the turbulent kinetic energy, is given by

$\kappa=\frac{\left\langle u_{i}^{\prime} u_{i}^{\prime}\right\rangle}{2}$

$v_{\mathrm{t}}$ is the eddy viscosity, $v$ the kinematic viscosity of the fluid and $d_{i j}$ the velocity strain

$d_{i j}=\frac{\partial u_{i}}{\partial x_{j}}+\frac{\partial u_{j}}{\partial x_{i}}$.

The dissipation rate of turbulent kinetic energy, $\epsilon$, is given by
Table 1

Model constants

\begin{tabular}{lllll}
\hline$c_{v}$ & $c_{\epsilon 1}$ & $c_{\epsilon 2}$ & $\sigma_{\kappa}$ & $\sigma_{\epsilon}$ \\
\hline 0.09 & 1.44 & 1.92 & 1.0 & 1.30 \\
\hline
\end{tabular}

$\epsilon=v\left\langle\frac{\partial u_{i}^{\prime}}{\partial x_{j}} \frac{\partial u_{i}^{\prime}}{\partial x_{j}}\right\rangle$

In the $\kappa-\epsilon$ model, dimensional arguments are invoked to give

$v_{\mathrm{t}}=c_{v} \frac{\kappa^{2}}{\epsilon}$

where $c_{v}$ is a model constant.

The turbulence parameters, $\kappa$ and $\epsilon$, are determined through the following transport equations

$\frac{D \kappa}{D t}=P-\epsilon+\frac{\partial}{\partial x_{i}}\left(\frac{v_{\mathrm{t}}}{\sigma_{\kappa}} \frac{\partial \kappa}{\partial x_{i}}\right)$

$\frac{D \epsilon}{D t}=\frac{\partial}{\partial x_{i}}\left(\frac{v_{\mathrm{t}}}{\sigma_{\epsilon}} \frac{\partial \epsilon}{\partial x_{i}}\right)+c_{\epsilon 1} \frac{\epsilon}{\kappa} P-c_{\epsilon 2} \frac{\epsilon^{2}}{\kappa}$

$P=v_{\mathrm{t}} \frac{\partial u_{i}}{\partial x_{i}}\left(\frac{\partial u_{i}}{\partial x_{j}}+\frac{\partial u_{j}}{\partial x_{i}}\right)$

where all the $c$ s and $\sigma$ s are model constants. Typical values of the empirical constants are shown in Table 1.

Clearly, the system of Eqs. (1)-(11) constitutes a closed system. The complete definition of the mathematical problem depends now only on the appropriate specification of the boundary conditions. At the wall, this is normally made with the help of wall functions. For the streamwise velocity component, $u$, for $\kappa$ and for $\epsilon$ we normally write

$\frac{u_{1}}{u_{\tau}}=\frac{1}{k} \ln \left(E_{1} y_{1}^{+}\right), \quad y_{1}^{+}=\frac{y_{1} u_{\tau}}{v}$

$\kappa=\frac{u_{\tau}^{2}}{\sqrt{c_{v}}}$

$\epsilon=\frac{u_{\tau}^{3}}{k y_{1}}$

where $u_{1}$ and $y_{1}$ should be evaluated in the fully turbulent region of the flow, $k$ is the von Karman constant $(=0.41), E_{1}$ is the linear coefficient of the law of the wall $(=9)$ and $u_{\tau}$ is the friction velocity. The above equations are derived upon the assumption of a local equilibrium condition and are valid for solid surface 
flows. In the next section we will extend these expressions to our case of interest.

\section{Asymptotic analysis}

Since the wall function formulation is supposed to hold in the fully turbulent region, we will solve the equations of motion, Eqs. (1)-(11), in a specific region of the flow.

Under this condition, the original equations of motion may undergo a series of simplifications. Depending on the local scales of the flow, asymptotic arguments can be evoked to evaluate the relative order of magnitude of the various terms in the motion equation. Further arguments show how terms which are supposed to locally dominate the solution can be selected to yield approximate equations with, preferably, analytical solutions. If the approximated equations are adequately chosen, one may then be capable of constructing a solution which is uniformly valid in the whole interval domain through successive matching of adjacent solutions.

The above ideas, very familiar to perturbation method users, can be given different degrees of mathematical formalism. For simple problems, simple rules and recipes can be devised to find solutions that can be matched in an appropriate overlap domain. For most of these simple rules the concept of overlap does not appear explicitly. However, matching is, by its nature, a comparison of two approximations in their domain of overlap.

To overcome the difficulties associated with the matching process, Kaplun [10] developed a theory based entirely on some formal properties of equations which aimed at characterizing them through their domains of validity. The theory naturally leads to a more fundamental understanding of the matching process and of the construction of the asymptotic expansions. The formal properties are established through passage of a limit process, the $\eta$-limit. Then, the concepts of formal domain, of intermediate equation, of principal equation and of formal domain of validity can be introduced. The operational details of the mathematical procedure are laid by five definitions, one Axiom and one Ansatz. Because of the heuristic nature of the Axiom and of the Ansatz, all results obtained through Kaplun's theory have to be experimentally validated. The absolute originality of the theory, however, always makes its application worthwhile.

An application of Kaplun's theory to the turbulent boundary layer was made by Cruz and Silva Freire [11]. The paper studied the asymptotic structure of both the velocity and the temperature turbulent boundary layers for attached and separating flows. An interesting result was the determination of two principal equations at points ord $\eta=$ ord $u_{\tau}^{2}$ and ord $\eta=$ ord $\left(1 / u_{\tau} R\right)$ of the formal validity domain. This result throws new light onto the asymptotic structure derived by Sychev and Sychev [12], giving a different interpretation to all three relevant length scales found there. In fact, what is said by the result is that it suffices to have two sets of approximate equations to cover the whole domain. Furthermore, these two sets are shown to overlap in an appropriate domain.

We will not repeat here the details of the analysis of Cruz and Silva Freire [11] which can be obtained directly from the original source. We just point out that all results were derived without appealing to any particular closure model; only asymptotic arguments were used. On this note, it must be said that the asymptotic structure remains the same as the considered $\kappa-\epsilon$ model. In fact, if the $\kappa$ and $\epsilon$ equations are considered in the analysis of Cruz and Silva Freire [11], it can be shown that the overlap domain defined by set (15) below remains unaltered.

The region where the turbulent effects dominate is defined by the domain

$D=\left\{\eta /\right.$ ord $\left.\left(1 / u_{\tau} R\right)<\operatorname{ord}(\eta)<\operatorname{ord}\left(u_{\tau}^{2}\right)\right\}$

where $\eta$ denotes the region of validity of the approximated equations, and $R$ denotes the Reynolds number. For more details concerning the nature of the stretching function $\eta$, we refer the reader to Kaplun [10], Cruz and Silva Freire [11], Meyer [13] or Silva Freire and Hirata [14].

The fully turbulent region, determined by Eq. (15), must be interpreted as the overlap domain of the two principal equations. Another result is that the presence of transpiration does not alter in any way domain (15). That means we should work with approximate equations that hold in that domain.

Passing the $\eta$-limit [11] with ord $(\eta)=\operatorname{ord}\left(u_{\tau}^{2}\right)$ into Eqs. (2)-(11), we get

$$
\begin{aligned}
& v_{\mathrm{w}} \frac{\partial u}{\partial y}=\frac{\partial}{\partial y}\left[c_{v} \frac{\kappa^{2}}{\epsilon} \frac{\partial u}{\partial y}\right] \\
& v_{\mathrm{w}} \frac{\partial \kappa}{\partial y}=v_{\mathrm{t}}\left(\frac{\partial u}{\partial y}\right)^{2}-\epsilon+\frac{\partial}{\partial y}\left(\frac{v_{\mathrm{t}}}{\sigma_{\kappa}} \frac{\partial \kappa}{\partial y}\right) \\
& v_{\mathrm{w}} \frac{\partial \epsilon}{\partial y}=c_{\epsilon} \frac{\epsilon}{\kappa} v_{\mathrm{t}}\left(\frac{\partial u}{\partial y}\right)^{2}-c_{\epsilon} \frac{\epsilon^{2}}{\kappa}+\frac{\partial}{\partial y}\left(\frac{v_{\mathrm{t}}}{\sigma_{\epsilon}} \frac{\partial \epsilon}{\partial y}\right) .
\end{aligned}
$$

These are the intermediate equations, in the sense of Kaplun, that hold in the fully turbulent region. The wall functions for $u, \kappa$ and $\epsilon$ will be constructed on their basis. 
To find a solution to Eqs. (16)-(18), we consider the flow quantities to be given by the following asymptotic expansions

$u=u_{\tau} u_{1}+v_{\mathrm{w}} u_{2}$

$\kappa=u_{\tau}^{2} \kappa_{1}+u_{\tau} v_{\mathrm{w}} \kappa_{2}+v_{\mathrm{w}}^{2} \kappa_{3}$

$\epsilon=u_{\tau}^{3} \epsilon_{1}+u_{\tau}^{2} v_{\mathrm{w}} \epsilon_{2}+u_{\tau} v_{\mathrm{w}}^{2} \epsilon_{3}$.

The first- and second-order of approximate equations can be found if we substitute Eqs. (19)-(21) into Eqs. (16)-(18) and collect the terms of the same order. The results are:

(a) first-order equations

$$
0=\frac{\partial}{\partial y}\left[c_{v} \frac{\kappa_{1}^{2}}{\epsilon_{1}} \frac{\partial u_{1}}{\partial y}\right]
$$

$$
0=c_{v} \frac{\kappa_{1}^{2}}{\epsilon_{1}}\left(\frac{\partial u_{1}}{\partial y}\right)^{2}-\epsilon_{1}+\frac{\partial}{\partial y}\left[\frac{c_{v}}{\sigma_{K}} \frac{\kappa_{1}^{2}}{\epsilon_{1}} \frac{\partial \kappa_{1}}{\partial y}\right]
$$

$$
0=c_{\epsilon 1} c_{v} \kappa_{1}\left(\frac{\partial u_{1}}{\partial y}\right)^{2}-c_{\epsilon 2} \frac{\epsilon_{1}^{2}}{\kappa_{1}}+\frac{\partial}{\partial y}\left[\frac{c_{v}}{\sigma_{\epsilon}} \frac{\kappa_{1}^{2}}{\epsilon_{1}} \frac{\partial \epsilon_{1}}{\partial y}\right]
$$

(b) second-order equations

$$
\begin{aligned}
\frac{\partial u_{1}}{\partial y} & =\frac{\partial}{\partial y}\left[\left(c_{v} \frac{\kappa_{1}^{2}}{\epsilon_{1}} \frac{\partial u_{2}}{\partial y}\right.\right. \\
& \left.\left.+\frac{1}{\epsilon_{1}}\left(2 \kappa_{1} \kappa_{2}-\frac{\epsilon_{2}}{\epsilon_{1}} \kappa_{1}^{2}\right) \frac{\partial u_{1}}{\partial y}\right)\right] \\
\frac{\partial \kappa_{1}}{\partial y} & =2 c_{v} \frac{\kappa_{1}^{2}}{\epsilon_{1}} \frac{\partial u_{1}}{\partial y} \frac{\partial u_{2}}{\partial y} \\
& +\frac{c_{v}}{\epsilon_{1}}\left(2 \kappa_{1} \kappa_{2}-\frac{\epsilon_{2}}{\epsilon_{1}} \kappa_{1}^{2}\right)\left(\frac{\partial u_{1}}{\partial y}\right)^{2}-\epsilon_{2} \\
& +\frac{c_{v}}{\sigma_{K}} \frac{\partial}{\partial y}\left[\left(\frac{\kappa_{1}^{2}}{\epsilon_{1}} \frac{\partial \kappa_{2}}{\partial y}\right.\right. \\
& \left.\left.+\frac{1}{\epsilon_{1}}\left(2 \kappa_{1} \kappa_{2}-\frac{\epsilon_{2}}{\epsilon_{1}} \kappa_{1}^{2}\right) \frac{\partial \kappa_{1}}{\partial y}\right)\right]
\end{aligned}
$$

$$
\begin{aligned}
\frac{\partial \epsilon_{1}}{\partial y} & =c_{v} c_{\epsilon 1}\left(2 \kappa_{1} \frac{\partial u_{1}}{\partial y} \frac{\partial u_{2}}{\partial y}\right. \\
& \left.+\left(2 \kappa_{2}-\frac{\epsilon_{2}}{\epsilon_{1}} \kappa_{1}\right)\left(\frac{\partial u_{1}}{\partial y}\right)^{2}\right) \\
& -c_{\epsilon 2} \frac{1}{\kappa_{1}}\left(2 \epsilon_{1} \epsilon_{2}-\frac{\kappa_{2}}{\kappa_{1}} \epsilon_{1}^{2}\right) \\
& +\frac{c_{v}}{\sigma_{\epsilon}} \frac{\partial}{\partial y}\left[\left(\frac{\kappa_{1}^{2}}{\epsilon_{1}} \frac{\partial \epsilon_{2}}{\partial y}\right.\right. \\
& \left.\left.+\frac{1}{\epsilon_{1}}\left(2 \kappa_{1} \kappa_{2}-\frac{\epsilon_{2}}{\epsilon_{1}} \kappa_{1}^{2}\right) \frac{\partial \epsilon_{1}}{\partial y}\right)\right] .
\end{aligned}
$$

The solution of the above equations is:

(a) first-order solution

$$
\begin{aligned}
& u_{1}=\frac{1}{k}\left(\ln y^{+}+A_{0}\right) \\
& \kappa_{1}=A \\
& \epsilon_{1}=\frac{B}{k y}
\end{aligned}
$$

(b) second-order solution

$$
u_{2}=\frac{1}{4 k^{2}}\left(\ln y^{+}+A_{0}\right)^{2}
$$

$\kappa_{2}=C \ln y^{+}+D$

$$
\epsilon_{2}=E \frac{\ln y^{+}}{y}+\frac{F}{y}
$$

where $y^{+}=y u_{\tau} / v, k$ is the von Karman constant and $A_{0}$ is a parameter that was shown to vary with the transpiration rate [17].

The constants $A, B, C, D, E$ and $F$ are given by

$$
A=\frac{1}{\sqrt{c_{v}}}=3.3
$$

$B=1$

$C=\frac{1}{k \sqrt{c_{v}}}=8.1$ 
$D=\frac{1}{k \sqrt{c_{v}}}\left(\frac{17}{2}-\sigma_{\epsilon}\right)=58.3$

$E=\frac{3}{k^{2}}=17.9$

$F=\frac{49-2 \sigma_{\epsilon}}{4 k^{2}}=69.6$

Please note that the values of these constants were determined exactly from the standard $\kappa-\epsilon$ model constants, which, in principle, cannot be said to be the best for the present class of problem. Thus, proper values for $A, B, C, D, E$ and $F$ should, necessarily, be determined by comparison with the experimental data. In the next two sections we will proceed to the experimental validation of Eqs. (20) and (21). This will be made in two ways. Firstly, we will verify if the experimental data conform to log-law expressions for $\kappa$ and $\epsilon$ such as those given by Eqs. (20) and (21). Secondly, a numerical implementation of these equations will be made for the prediction of velocity profiles and the skin-friction coefficient.

The velocity solution given by Eqs. (19), (28) and (31) dispenses any kind of validation test since it consists of a well-known result. This equation has been thoroughly tested over the last thirty years, being of undisputed validity today. It can be arrived at by either dimensional arguments or mathematical deductions that use simple algebraic turbulence models $[15,16]$.

The velocity solution is given by

$u=\frac{u_{\tau}}{k}\left(\ln y^{+}+A_{0}\right)+\frac{v_{\mathrm{w}}}{4 k^{2}}\left(\ln y^{+}+A_{0}\right)^{2}$

where $A_{0}$ was shown by Silva Freire [17] to vary with the transpiration rate according to

$A_{0}=k\left(5.0-512 \frac{v_{\mathrm{w}}}{U_{\infty}}\right)$.

An extension of Eq. (40) for the outer part of the flow can readily be obtained if an appropriate wake function is added to it. This wake function is also shown to depend on the transpiration rate [17]. The resulting skin-friction equation is much less sensitive than other formulations to small variations in the flow parameters, providing very reliable results.

For compressible transpired boundary layer flows, the law of the wall is cast in the form of an elliptic integral [18], which makes it difficult to be handled algebraically. However, Silva Freire [19] has shown that a direct application of a van Driest transformation to Eqs. (40) and (41) provides very good results in the prediction of flow velocity and skin-friction.

\section{Experimental and numerical validation}

From a full consideration of more than 200 boundary layer developments with transpiration, Square [20] recommends as a basic test case the flows measured by Andersen et al. [9]. These data are, according to the former author, the only ones available in literature where redundant measurements of the skin-friction coefficient were made and for this reason are relatively free of error. Andersen et al. [9] proposed to determine the wall shear stress by measuring the shear stress away from the wall and use the integrated boundary layer equations to extrapolate the results to the wall. This new technique was supposed to give 'independence' to the data.

The principal objective of the work of Andersen et al. [9] was to provide measurements for transpired boundary layers in adverse pressure gradients. A secondary motivation was to repeat some of Simpson et al.'s [21] constant pressure flow since, during some exploratory tests, it had been found that the mean velocity data of Simpson et al. [21] obtained through flattened Pitot tubes differed appreciably from profiles obtained through hot-wire anemometry. Thus, because the measurements for zero pressure gradient boundary layers were taken at an early age and through Pitot tubes, no data on turbulent quantities were presented. This fact seriously compromises the experimental assessment of constants $C, D, E$ and $F$. After the initial tests of Simpson et al. [21], the authors that followed concentrated on more complex flow geometries, leaving the zero pressure gradient results as they were.

In Andersen et al.'s report, twenty runs are listed. After a specific section where a run summary of some relevant parameters is presented, the profile tables containing a detailed list of the flow conditions including boundary conditions, skin-friction coefficients and integral parameters follow. It is important to note that no turbulent quantity is given in tabulated form; they are presented in graphical form in the main body of the work. In particular, no turbulent quantity is presented for flows without external adverse pressure gradient. For this reason, only three runs could be considered for validation of the data on turbulent quantities; those whose flows were subjected to a constant injection rate and to a low adverse pressure gradient. To all the other runs the flows were either subjected to a distributed injection rate, or to a very strong adverse pressure gradient. Thus, strictly speaking, none of the flow conditions of Andersen et al. apply for consideration here. However, since the present authors were not capable of locating any other source of data which could be of use, they have decided to consider for validation of the theory the three experimental runs which most approached the recognized theoretical conditions.

The general experimental conditions are shown in 
Table 2

Experimental flow conditions

\begin{tabular}{llrl}
\hline Run & $U[\mathrm{ft} / \mathrm{s}]$ & $F$ & \multicolumn{1}{l}{$m$} \\
\hline $102171-1$ & 21.33 & 0.004 & -0.15 \\
$101371-2$ & 21.32 & 0.002 & -0.15 \\
$121671-3$ & 21.29 & -0.002 & -0.15 \\
\hline
\end{tabular}

Table 2, where $F$ stands for injection rate, $m$ is the exponential coefficient in the adverse pressure gradient term, $\left(U \propto x^{m}\right)$.

To verify the logarithmic form of $\kappa$, we re-write Eq. (19) as

$\Gamma=C \ln y^{+}+D$

where

$\Gamma=\frac{\kappa-u_{\tau}^{2} A}{u_{\tau} v_{\mathrm{w}}}$

The experimental results plotted as $\Gamma$ against $y^{+}$are shown in Fig. 1. The presence of a logarithmic region is clearly illustrated, thus confirming the form obtained for Eq. (42). In fact, the injection data seem to indicate that parameters $C$ and $D$ are presumably constant since the two curves collapse nicely. The suction results, on the other hand, give completely different values for parameters $C$ and $D$.

In truth, even casual observation of the experimental data shows that expressions (13) and (14) cannot provide a good description for the boundary conditions.

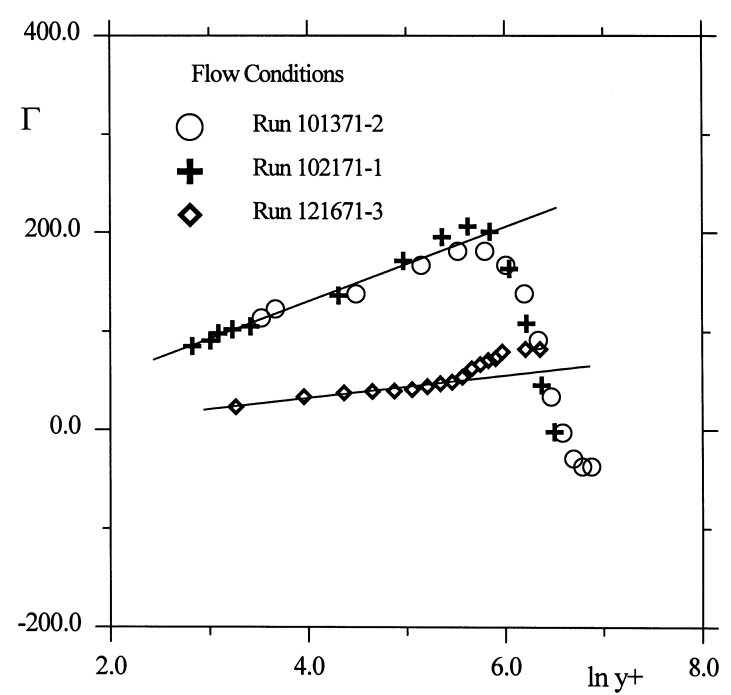

Fig. 1. The behaviour of $\Gamma$ according to the data of Andersen et al. [9].
The difficulty here is that $\kappa$ is seen to increase with an increase in injection rate whereas $u_{\tau}$ is seen to decrease. When section is applied to the flow, the inverse behaviour is observed; an increase in suction decreases $\kappa$ and increases $u_{\tau}$. The conclusion is that Eq. (13) cannot reconcile this experimental fact. The higher-order correction term is, therefore, crucial for a good representation of the flow field.

Following the mean velocity trend, we must expect $C$ and $D$ to vary with the injection rate and, indeed, even the small quantity of data gathered here appears to indicate this. Any definitive functional expression for their behaviour should, however, only be advanced as further data on $\kappa$ becomes available in the literature.

Since $\epsilon$ is not a directly measurable quantity, its experimental assessment is difficult to be made. Nevertheless, its behaviour can be indirectly studied through equation

$\epsilon=C_{D} \frac{\kappa^{3 / 2}}{l_{\mathrm{c}}}$

where the constant $C_{D}$ is a model constant.

This procedure is always uncertain, making it difficult to draw any firm conclusion about the values of the constants $E$ and $F$; however, it should disclose any logarithmic behaviour of $\epsilon$.

The $\epsilon$ profile will be represented in the similarity variables $\xi$ and $y^{+}$, defined as

$\Xi=k\left(E \ln y^{+}+F\right)$

where

$\Xi=\frac{\epsilon(k y)-u_{\tau}^{3} B}{u_{\tau}^{2} v_{\mathrm{w}}}$

The experimental results plotted as $\xi$ against $y^{+}$are shown in Fig. 2.

It is no surprise that the assessed experimental values are different from the theoretical ones since, as earlier explained, the experimental flow conditions do not exactly reproduce the theoretical conditions. However, it is clear from Figs. 1 and 2 that both $\kappa$ and $\epsilon$ do present a logarithmic behaviour in a certain region of the flow. As mentioned before the theoretical values were evaluated according to the classical $\kappa-\epsilon$ model constants and these, in principle, are not supposed to hold for transpired flows.

Since Simpson et al. [21] applied suction from the leading edge of the test surface, their boundary layer was very thin closely approximating the asymptotic state. Under this condition, much of the velocity fluctuations are removed, resulting in a boundary layer which is in a near state of relaminarization. For this 


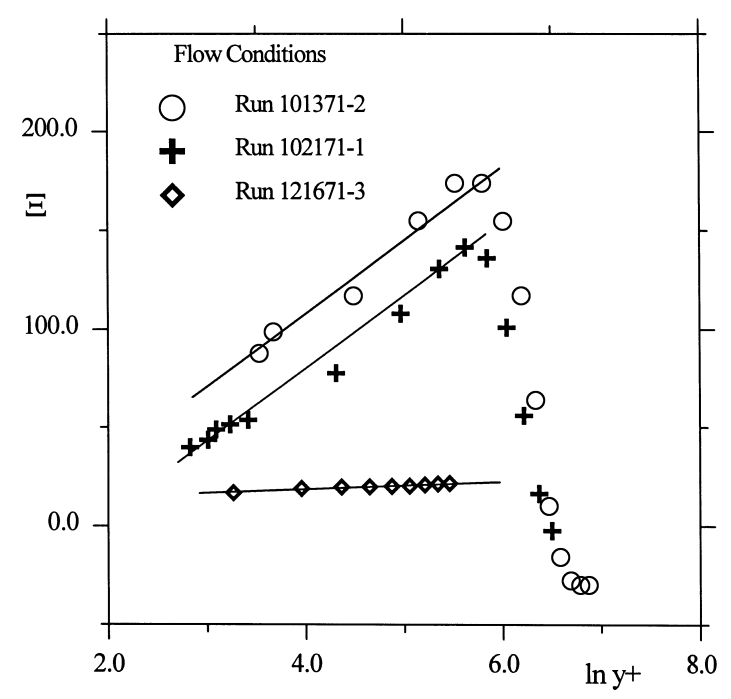

Fig. 2. The behaviour of $\xi$ according to the data of Andersen et al. [9].

reason, some of the results for the suction data differ appreciably from the blowing data.

To proceed to an independent assessment of the theory, Eqs. (19)-(21) will be implemented in a numerical representation of the flow field. Then, the calculated velocity and skin-friction profiles will be compared with the experimental data of Andersen [9] for flows subjected to zero pressure gradients. We point out that the implemented numerical values of the constants $A$ to $F$ will be the theoretical values provided by Eqs. (34)-(39).

The numerical results were generated through an especially constructed computer code. Using the boundary layer approximations, the motion equations were disceretized by way of a fully implicit finite difference scheme [22] on a non-uniformly spaced grid in the transversal direction. The finite difference discretization results in a system of $4 N$ couple non-linear algebraic equations, which had to be solved at each streamwise station in a space-marching procedure for parabolic partial differential equations.

The difference equations were iterated according to

$\mathbf{A}^{k}\left(\mathbf{u}^{k+1}-\mathbf{u}^{k}\right)=-\mathbf{r}^{k}$

where matrix $\mathbf{A}^{k}$ is the linearized equation matrix (LEM) formed from values determined in the $k$ th nonlinear iteration, the vector $\mathbf{u}^{k}$ represents the variables of the system of linear algebraic equations, and $\mathbf{r}^{k}$ is the residue of the equations at the $k$ th iteration. For the applied finite difference method, the LEM has a block tri-diagonal structure, where the elements of the block matrices are determined by a modified Newton method. A simplified Jacobian was used to form the iteration matrix by keeping constant some of the coefficients. The inversion of each $4 \times 4$ element matrix was made by a LU decomposition method. The simultaneously coupled solution of all equations significantly improves the robustness and efficiency of the numerical scheme for flow conditions at high Reynolds numbers.

An adaptive algebraic grid generator was used to redistribute the grid points at each position after a preliminary solution had been obtained. Basically, a geometric progression extending from the nearest to the wall grid point to the most external grid point was implemented.

The point where the wall boundary conditions were implemented was chosen according to domain (15). For most flows of interest this can be translated into

$D=\left\{y^{+} / 11<y^{+}<110\right\}$.

Here we have taken $y^{+}=y u_{\tau} / v=40$. The upper grid limit was taken as $3 \delta$.

The streamwise stepsize was made a fraction of the local boundary layer momentum thickness. A systematic study of the influence of the computational parameters on the solution has been carried out by one of the present authors [23] and is reported elsewhere.

The numerical predictions were compared with the experiments shown in Table 3. These runs cover a large range of injection rates and, unlike those of Table 2, are for flows with zero external pressure gradients.

Figs. 3-6 show the numerical predictions for the skin-friction coefficient as compared with the experimental data of Andersen et al. [9] and the standard $\kappa-$ $\epsilon$ model formulation. As can be seen, the performance of the present formulation is remarkable even for the highest transpiration rate $(=0.008)$. The standard model always overpredicted the skin-friction coefficient, even for the higher values of the local Reynolds number based on the momentum thickness. This fact has also been observed by Rodi and Schreurer [24], who argue that the standard model predicts an increase of the near wall slope of the length scale with blowing, whereas the experiments do not show such a dependence. For $F=0.008$ the standard model did not converge. For low Reynolds numbers, the predictions

Table 3

Experimental flow conditions

\begin{tabular}{llll}
\hline Run & $U[\mathrm{ft} / \mathrm{s}]$ & $F$ & $m$ \\
\hline $100571-1$ & 31.17 & 0.001 & 0.0 \\
$090171-2$ & 31.38 & 0.002 & 0.0 \\
$090871-2$ & 31.40 & 0.00375 & 0.0 \\
$092271-5$ & 31.51 & 0.008 & 0.0 \\
\hline
\end{tabular}




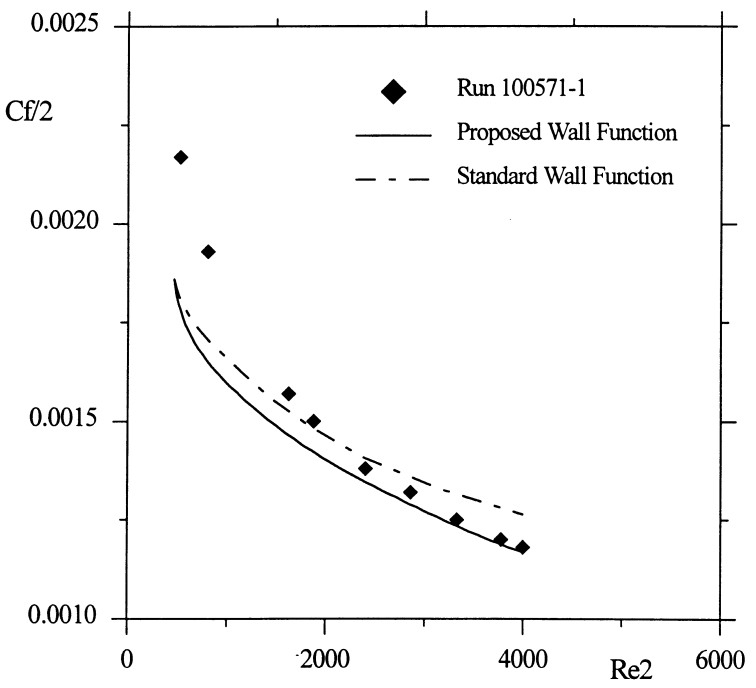

Fig. 3. Skin-friction coefficient prediction, $F=0.001$.

departed from the experimental data in view of the uncertainties related to the specification of the initial conditions for $\kappa$ and for $\epsilon$. Indeed, as said before, no data for $\kappa$ were presented in Andersen et al. [9] for the zero pressure gradient flow cases. The present authors were then forced to specify an arbitrary and uniform value of $\kappa / U_{\infty}^{2}(=0.01)$ as an initial condition. The result was that only as the flow had progressed some distance downstream the numerical results started to approach the experimental data asymptotically.

A typical velocity profile is shown in Fig. 7. the good agreement of the presently proposed theory can, again, by fully appreciated here. The almost exact reproduction of the experimental data by the new for-

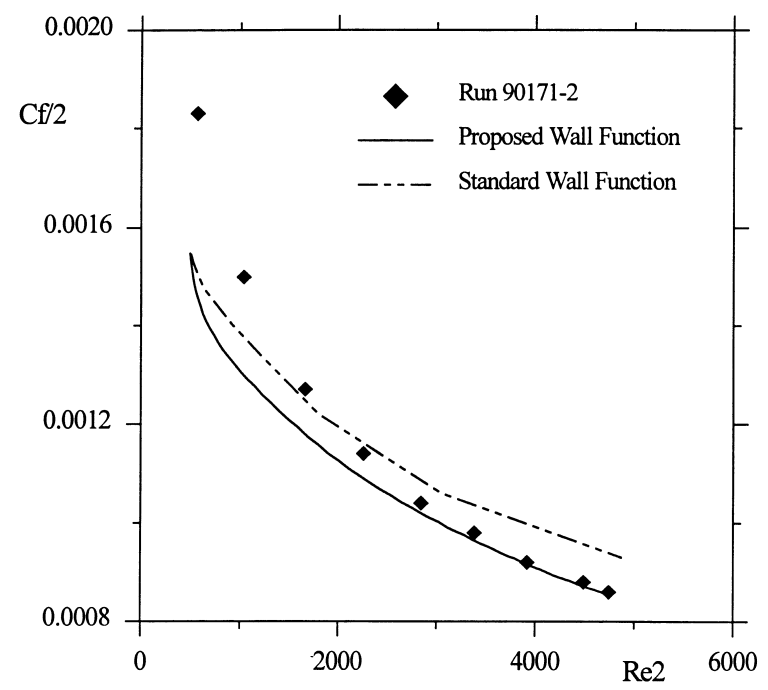

Fig. 4. Skin-friction coefficient prediction, $F=0.002$.

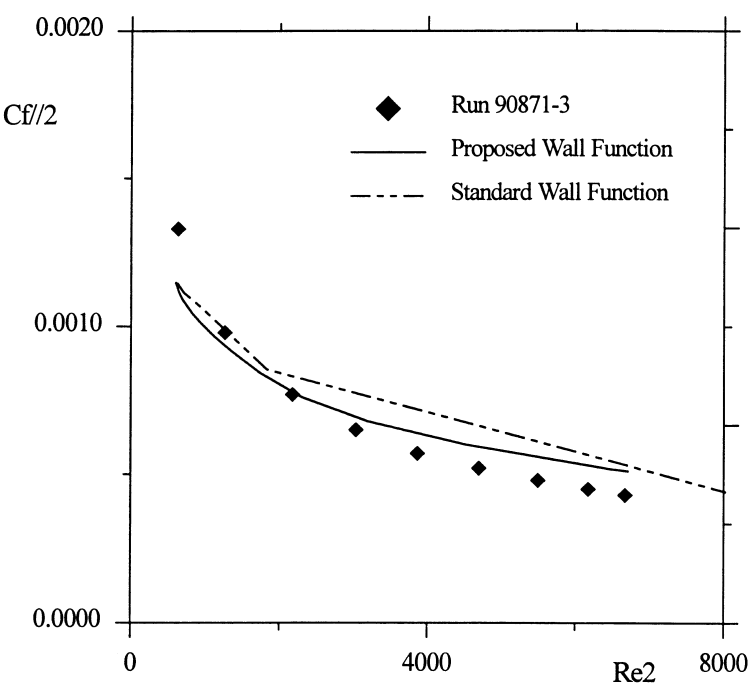

Fig. 5. Skin-friction coefficient prediction, $F=0.00375$.

mulation is, indeed, very impressive - the law of the wall and the law of the wake are accurately predicted.

\section{Final remarks}

Since the standard $\kappa-\epsilon$ model does not apply to flows or flow regions where the local Reynolds number is small, it cannot be applied to the viscous near wall region. A popular approach to computing turbulent flow has been to bridge this region by universal wall functions. The present work aimed at finding a highorder solution for $u, \kappa$ and $\epsilon$ that have an explicit dependence on the transpiration rate and can be used

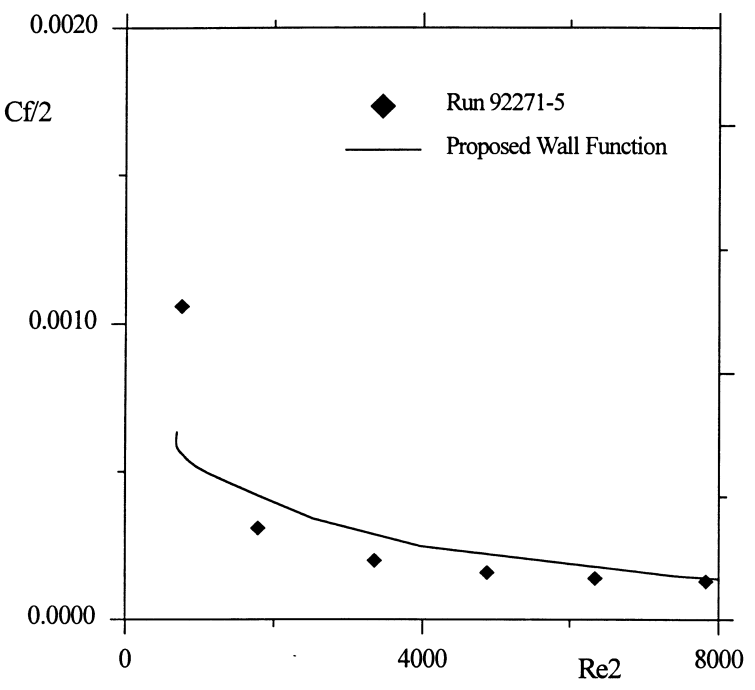

Fig. 6. Skin-friction coefficient prediction, $F=0.008$. 


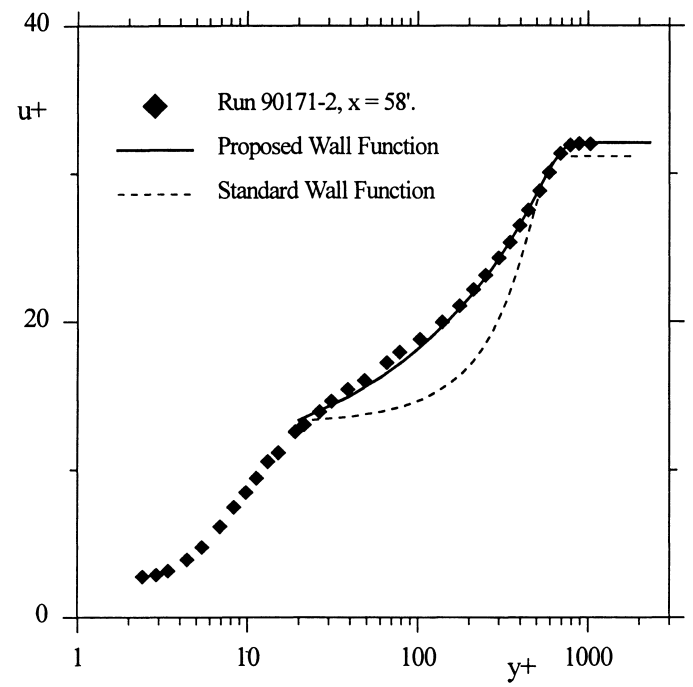

Fig. 7. Velocity profiles for $F=0.002, R e 2=3384$.

as wall functions. These new solutions should greatly improve the performance of the $\kappa-\epsilon$ model for transpired flows, in particular for flows with strong transpiration rates.

Keeping that in mind, a consistent asymptotic solution for the $\kappa-\epsilon$ model for incompressible transpired turbulent boundary layers was developed. The solution unveils, for the first time, the logarithmic behaviour of $\kappa$ and of $\epsilon$ in the fully turbulent region of the flow. A numerical implementation of the new expressions shows that they conform well to the experimental data, giving good predictions for the velocity profile and the skin-friction equation.

\section{Acknowledgements}

This work was financially supported by the CNPq through Grant No. 350183/93-7. The authors M.R.A. and J.S. are grateful, respectively, to CAPES (Ministry of Education) and to the CNPq (Ministry of Science and Technology) for the award of research scholarships.

\section{References}

[1] H.S. Mickley, R.S. Davis, Momentum transfer for flow over a flat plate with blowing, NACA TN 4017, 1957.

[2] A.P. Silva Freire, D.O.A. Cruz, C.C. Pellegrini, Velocity and temperature distribution in compressible turbulent boundary layers with heat and mass transfer, Int. J. Heat Mass Transfer 13 (1995) 2507-2515.

[3] R.A. Antonia, L. Fulachier, Topology of a turbulent boundary layer with and without wall suction, J. Fluid Mechanics 198 (1989) 429-451.
[4] R.A. Antonia, D.K. Bisset, L. Fulachier, F. Anselmet, Effect of wall suction on bursting in a turbulent boundary layer, Phys. Fluids A 2 (1990) 1241-1247.

[5] L. Fulachier, F. Anselmet, T. Benabid, R.A. Antonia, Normalization for a turbulent boundary layer with wall suction, Phys. Fluids A 3 (1991) 233-235.

[6] T.J. Dahm, R.M. Kendall, Comments on inner region of transpired turbulent boundary layers, AIAA J. 6 (1968) 1822-1824.

[7] N. Shima, Prediction of turbulent boundary layers with a second-moment closure: part I-effects of periodic pressure gradient, wall transpiration, and free-stream turbulence, J. Fluids Eng. 115 (1993) 56-63.

[8] B.E. Launder, N. Shima, Second-moment closure for the near-wall sublayer: development and application, AIAA J. 10 (1989) 1319-1325.

[9] P.S. Andersen, W.M. Kays, R.J. Moffat, Turbulent boundary layer on a porous plate: an experimental study of the fluid mechanics for adverse free-stream pressure gradients, Report No. HMT-15, Thermosciences Division, Department of Mechanical Engineering, Stanford University, 1972.

[10] S. Kaplun, Fluid Mechanics and Singular Perturbation, Academic Press, New York, 1967.

[11] D.O.A. Cruz, A.P. Silva Freire, On single limits and the asymptotic behaviour of separating turbulent boundary layers, Int. J. Heat Mass Transfer 41 (1998) 2097-2111.

[12] V.V. Sychev, V.V. Sychev, On turbulent boundary layer structure, P.M.M. U.S.S.R. 51 (1987) 462-467.

[13] R.E. Meyer, On the approximation of double limits by single limits and the Kaplun extension theorem, J. Inst. Maths Applics. 3 (1967) 245-249.

[14] A.P. Silva Freire, M.H. Hirata, Approximate solution to singular perturbation problems: the intermediate variable technique, J. of Mathematical Analysis and Applications 145 (1990) 241-253.

[15] T.N. Stevenson, A modified defect law for turbulent boundary layers with suction or injection, Aero Rep. No. 170, Cranfield College of Aero, 1963.

[16] T.N. Stevenson, Experiments on injection into an incompressible turbulent boundary layer, Aero Rep. No. 177, Cranfield College of Aero, 1963.

[17] A.P. Silva Freire, An asymptotic solution for transpired incompressible turbulent boundary layers, Int. J. Heat Mass Transfer 31 (1988) 1011-1021.

[18] L.C. Squire, A law of the wall for compressible turbulent boundary layers with air injection, J. Fluid Mechanics 37 (1969) 449-456.

[19] A.P. Silva Freire, An extension of the transpired skinfriction equation to compressible turbulent boundary layers, Int. J. Heat Mass Transfer 31 (1988) 2395-2398.

[20] L.C. Squire, Turbulent boundary layers with suction or injection. Conference on Data and Computation for Complex Flows, Report of Evaluator, Stanford, 1980.

[21] R.L. Simpson, W.M. Kays, R.J. Moffat, The turbulent boundary layer on a porous plate: an experimental study of the fluid dynamics with injection and suction, Report No. HMT-2, Thermosciences Division, Department of Mechanical Engineering, Stanford University, 1967. 
[22] D.A. Anderson, J.C. Tannihill, R.H. Pletcher, Computational Fluid Mechanics and Heat Transfer, Hemisphere Publishing, Washington, DC, 1984.

[23] J. Su, An adaptive nonlinear coupled solution procedure for turbulent boundary layer equations with lowReynolds number two-equation models, in: Proceedings of the 4th North-Northeastern Congress on Mechanical Engineering (IV-CEM-NNE), Recife, Brazil, 1996.

[24] W. Rodi, G. Schreurer, Calculation of heat transfer in turbulent transpired boundary layers, in: Proceedings of the 7th International Heat Transfer Conference, Munich, 1982. 